\title{
ON SERIAL POSETS WITH POSITIVE-DEFINITE QUADRATIC TITS FORM
}

\author{
V. M. Bondarenko ${ }^{1}$ and M. V. Stepochkina ${ }^{2}$
}

UDC 512.647.2+512.562

\begin{abstract}
We describe all serial posets with positive-definite quadratic Tits form and prove that any poset of order greater than 7 with positive-definite Tits form is serial.
\end{abstract}

Quadratic forms are encountered in the solution of various problems in algebra, geometry, the theory of differential and integral equations, operator theory, and other fields of mathematics (see, e.g., [1-26]). Among them, an important role is played by quadratic Tits forms for oriented graphs, posets, algebras, etc. In the present paper, we consider exactly these forms.

\section{Formulation of the Main Result}

First, recall some definitions.

Let $S$ be a finite or an infinite poset. We say that $S$ is the sum of its subsets $A_{1}, \ldots, A_{s}$ and write $S=$ $A_{1}+\ldots+A_{s}$ if $S=\cup_{i=1}^{s} A_{i}$ and $A_{i} \cap A_{j}=\varnothing$ for $i \neq j$. If all elements of different terms are always incomparable, then $S$ is called the direct sum of the indicated subsets. Further, according to [27], the sum $S=$ $A_{1}+\ldots+A_{s}$ is called one-sided if (up to enumeration of terms) one has $i<j$ whenever there exist elements $b \in A_{i}$ and $c \in A_{j}$ for $i \neq j$ such that $b<c$. According to [27], the sum $S=A_{1}+\ldots+A_{s}$ is called minimax if it follows from the relation $x<y$, where $x$ and $y$ belong to different terms, that $x$ and $y$ are, respectively, minimal and maximal elements of the set $S$. Formally, a direct sum is minimax. Nevertheless, considering minimax sums in what follows, we always assume for convenience that they are not direct.

A subset of a poset $S$ is understood as a complete partially ordered subset, i.e., a partial order on it is induced by a partial order on $S$.

The form $q_{S}(z): \mathbb{Z}_{0}^{S \cup 0} \rightarrow \mathbb{Z}$ defined by the equality

$$
q_{S}(z)=z_{0}^{2}+\sum_{i \in S} z_{i}^{2}+\sum_{i<j, i, j \in S} z_{i} z_{j}-z_{0} \sum_{i \in S} z_{i}
$$

where $\mathbb{Z}_{0}^{S \cup 0}$ is a subset of the Cartesian product $\mathbb{Z}^{S \cup 0}=\left\{\left(z_{i}\right), i \in S \cup 0\right\}$ that consists of all vectors with finitely many nonzero coordinates (if $S$ is finite, then $\mathbb{Z}_{0}^{S \cup 0}=\mathbb{Z}^{S \cup 0}$ ), is called a quadratic Tits form of the poset $S$.

We now formulate the main result.

A finite or an infinite poset $S$ with positive-definite Tits form is called serial if, for any natural $m$, there exists a poset $T$ for which the following conditions are satisfied:

(a) $S$ is a subset of $T$;

(b) $|T \backslash S|=m$;

(c) the Tits form of the set $T$ is positive definite.

\footnotetext{
${ }^{1}$ Institute of Mathematics, Ukrainian National Academy of Sciences, Kyiv.

${ }^{2}$ Shevchenko Kyiv National University, Kyiv.
}

Translated from Neliniini Kolyvannya, Vol. 9, No. 3, pp. 320-325, July-September, 2006. Original article submitted April 5, 2006. 
Theorem 1. Any poset of order greater than 7 with positive-definite Tits form is serial.

In the process of the proof of the theorem, we indicate the explicit form of serial posets.

\section{Structure of Serial Posets}

We call a poset with a unique pair of incomparable elements a quasichain (a chain is an arbitrary linearly ordered set). The maximum number of pairwise-incomparable elements of a poset is called the width of the poset.

Let us prove the following theorem:

Theorem 2. A poset $S$ with positive-definite Tits form is serial if and only if one of the following conditions is satisfied:

(1) S is a direct sum of two chains;

(2) S is a one-sided minimax sum of two chains;

(3) $S$ is a direct sum of a chain and a quasichain.

Note that the chains in conditions (1) and (3) can be empty.

Geometrically, conditions (1)-(3) have the form

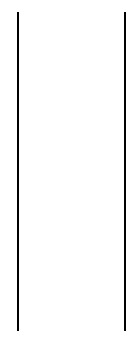

(1)

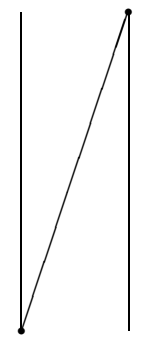

(2)

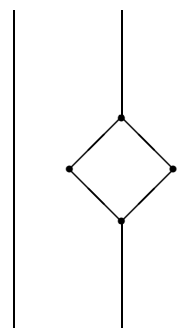

$(3)$

(here, the vertical lines are chains and the inclined segments do not contain intermediate points).

In fact, this theorem follows from the results of [27]. Namely, according to the main theorem of [27], an infinite poset has a positive-definite Tits form if and only if it has the form (1), (2), or (3). This means that Theorem 2 is true for infinite posets. This implies that Theorem 1 is also true for infinite posets because, in a poset of the form (1), (2), or (3), one can easily indicate a point such that, after the replacement of this point by a chain of $m$ points, a new poset has the same form.

In the proof of the theorem in [27], a certain list of posets for which the Tits form is not positive definite was used. Since this list is finite and consists of only finite posets, this means that there exists a certain natural number $N$ such that any finite poset of order greater than $N$ with positive-definite Tits form has the form (1), (2), or (3). This yields Theorem 2 for finite posets (see the explanation for infinite posets). Note that to prove Theorem 1 for finite posets is to show that the number 7 may be taken as $N$.

In view of Theorem 2, it is easy to see that a poset of order $n \leq 7$ with positive-definite Tits form can be not serial. For example, the poset $T=\left\{a, b_{1}, b_{2}, c_{1}, c_{2} \mid b_{1}<b_{2}, c_{1}<c_{2}\right\}$ (of order 5) is not serial. 


\section{Proof of Theorem 1 (for Finite Posets)}

In the proof of the theorem, we use the notion of ( $\min , \max$ )-equivalence of posets introduced in [28].

Recall the definition of this equivalence.

Let $S$ be a poset and let $a$ be its minimal (respectively, maximal) element. For elements $x, y \in S$, we write $x \gtrless y$ if $x$ and $y$ are incomparable. Denote the set of elements $x \in S$ incomparable with a fixed element $a \in S$ by $S^{\rtimes}(a)$. We always identify one-element subsets of $S$ with the elements themselves.

Let $S_{a}^{\uparrow}$ (respectively, $S_{a}^{\downarrow}$ ) denote the poset thus defined. As an ordinary set (i.e., regardless of a partial order), this is $S$; moreover, the partial order on $S \backslash a$ is preserved, $a$ is a maximal (respectively, minimal) element, and $a>x$ (respectively, $a<x$ ) if and only if $x \in S^{\ngtr}(a)$.

Further, we write $S_{x y}^{\uparrow \uparrow}$ instead of $\left(S_{x}^{\uparrow}\right)_{y}^{\uparrow}$, etc.

Let $S$ and $T$ be posets satisfying the condition $S=T$ as ordinary sets. We call them (min, max)-equivalent and write $T \cong_{(\min , \max )} S$ if

$$
T=S_{x_{1} x_{2} \ldots x_{p}}^{\varepsilon_{1} \varepsilon_{2} \ldots \varepsilon_{p}} \quad(p \geq 0)
$$

where $\varepsilon_{i} \in\{\uparrow, \downarrow\}$ and, for each $i=1, \ldots, p, x_{i}$ is a minimal (respectively, maximal) element of $S_{x_{1} x_{2} \ldots \varepsilon_{i-1}}^{\varepsilon_{1} \varepsilon_{2} \ldots \varepsilon_{i-1}}$ if $\varepsilon_{i}=\uparrow$ (respectively, $\varepsilon_{i}=\downarrow$ ); note that $x_{1}, x_{2}, \ldots, x_{p}$ are not necessarily different. For $p=0$, we assume that $T=S$.

In the case where all arrows in this notation are directed upward (respectively, downward), the posets $S$ and $T$ are called min-equivalent (respectively, max-equivalent). Note that any (min, max)-equivalent posets are both min-equivalent and max-equivalent. However, we neither prove this statement nor use it in the present paper.

It was proved in [28] that (min, max)-equivalent posets have equivalent Tits forms. Therefore, it is quite natural to study posets with positive-definite Tits form up to this equivalence.

The statement presented below follows directly from the definition of (min, max)-equivalence.

Proposition 1. Suppose that $T_{1}$ is the set of all minimal elements of a poset $T$ of order $n$ and (inductively) $T_{i}, \quad i>1$, is the set of all minimal elements of $T \backslash\left(\cup_{j=1}^{i-1} T_{j}\right)$; the notation $h(x)=i$ for $x \in T$ means that $x \in T_{i}$. Then, for any sequence without repetitions $\left(x_{1}, x_{2}, \ldots, x_{n}\right)$ such that $h\left(x_{1}\right) \leq h\left(x_{2}\right) \leq \ldots \leq h\left(x_{n}\right)$, the relation $T^{\prime}=T_{x_{1} x_{2} \ldots x_{n}}^{\uparrow \uparrow}$ is meaningful and, moreover, $T^{\prime}=T$.

We now pass to the proof of Theorem 1 for finite posets (for infinite posets, see the previous section). By virtue of Theorem 2, it suffices to show that any poset of order greater than 7 with positive-definite Tits form has the form (1), (2), or (3).

Denote the width of a poset $X$ (the maximal number of its pairwise-incomparable elements) by $w(X)$.

Let $S$ be a (finite) poset. We fix a certain maximal element $a$ in $S$ and denote the set of all elements $x \in S$ such that $x<a$ by $S(a)$. For $S(a)$, we fix the sequence $\left(y_{1}, y_{2}, \ldots, y_{s}\right)$ indicated in Proposition 1 and consider the poset $T=S_{y_{1} y_{2} \ldots y_{s}}^{\uparrow \uparrow \ldots}$. It is obvious that the element $a$ in $T$ is both minimal and maximal.

Since the Tits form $q(z)$ of a poset that consists of four pairwise-incomparable elements, say, 1, 2, 3, and 4 , is not positive definite $\left(q(z)=0\right.$ for $z_{0}=2$ and $\left.z_{1}=z_{2}=z_{3}=z_{4}=1\right)$, the case $w(T) \geq 4$ is impossible, and, hence, $w(T) \leq 3$. Therefore, it is obvious that the poset $P=T_{a}^{\uparrow}$ has the width $w \leq 2$.

Thus, we have proved that the poset $S$ is min-equivalent to a certain poset $P$ of width $2: P=S_{y_{1} y_{2} \ldots y_{s} a}^{\uparrow}$. Then $S=P_{a y_{s} \ldots y_{2} y_{1}}^{\downarrow \downarrow \ldots \downarrow}$ (because $X_{y y}^{\uparrow \downarrow}=X$ and $X_{z z}^{\downarrow \uparrow}=X$ for an arbitrary minimal element $y$ and an arbitrary maximal element $z$ of any poset $X$ ). Using the results of Sec. 2, we can complete the proof of Theorem 1 according to the following scheme:

I. Show that any poset of a set $S$ of width $w \leq 2$ and order $n>7$ with positive-definite Tits form has the form (1), (2), or (3) (in the last case, the chain that is the direct summand must be empty because otherwise $w=3$ ). 
II. Show that if a poset $S$ of width $w \leq 3$ and order $n>7$ has the form (1), (2), or (3) and $x$ is a maximal element of $S$, then $S_{x}^{\downarrow}$ is either a poset of width $w^{\prime} \leq 2$ [in this case, according to I, it has the form (1), (2), or (3)] or a poset of width 3 that has the form (3).

The statement required by step I was proved in [29].

We now proceed to step II.

Let $S$ be a poset of width $w \leq 3$ and order $n>7$ that has the form (1), (2), or (3). In the second case, for definiteness, we assume that the minimal element of the first chain is smaller than the maximal element of the second chain. Let $x$ be the maximal element of $S$ and let $T=S_{x}^{\downarrow}$. Then the following assertions are true:

(1.1) if $S$ has the form (1), then $w(T) \leq 2$ [namely, $T$ also has the form (1)];

(2.1) if $S$ has the form (2) and $x$ belongs to the first chain, then $w(T) \leq 2$ [namely, $T$ also has the form (2)];

(2.2) if $S$ has the form (2) and $x$ belongs to the second chain, then $T$ has the form (3) (both cases $w(T)=2$ and $w(T)=3$ are possible here);

(3.1) if $S$ has the form (3) and $x$ belongs to the chain, then $T$ has the form (3) (both cases $w(T)=2$ and $w(T)=3$ are possible here);

(3.2) if $S$ has the form (3), $x$ belongs to the quasichain, and the quasichain has a single maximal element, then $w(T)=3$ and $T$ has the form (3);

(3.3) if $S$ has the form (3), $x$ belongs to the quasichain, and the quasichain has two maximal elements, then $w(T) \leq 2$ [namely, $T$ also has the form (2)].

Theorem 1 is proved.

Note that the statement required in step II is also true for $n \leq 7$ (the proof is analogous).

\section{REFERENCES}

1. Yu. A. Drozd, “The Coxeter transformations and representations of posets," Funkts. Anal. Prilozhen., 8, 34-42 (1974).

2. Yu. A. Mitropol'skii, A. M. Samoilenko, and V. L. Kulik, "Application of quadratic forms to the investigation of systems of linear differential equations," Differents. Uravn., 21, No. 5, 776-788 (1985).

3. A. N. Kochubei, "Fundamental solutions of pseudodifferential equations related to $p$-adic quadratic forms," Izv. Ros. Akad. Nauk, 62, No. 6, 103-124 (1998).

4. P. Gabriel, "Unzerlegbare Darstellungen," Manuscr. Math., 6, 71-103, 309 (1972).

5. S. Brenner, "Quivers with commutativity conditions and some phenomenology of forms," Proceedings of the International Conference on Representations of Algebras, Paper No. 5, Carleton University, Ottawa (1974).

6. K. Bongartz, "Algebras and quadratic forms," J. London Math. Soc., 28, No. 3, 461-469 (1983).

7. C. M. Ringel, Tame Algebras and Integral Quadratic Forms, Springer, Berlin (1984).

8. M. G. Crandall, "Semidifferentials, quadratic forms and fully nonlinear elliptic equations of second order," Ann. Inst. H. Poincaré, Anal. Non Linéare, 6, No. 6, 419-435 (1989).

9. I. Corovei, "Some functional equations connected with quadratic forms," Anal. Numér. Théor. Approxim., 19, No. 2, 123-127 (1990).

10. I. Al-Naggar and D. B. Pearson, "Quadratic forms and solutions of the Schrödinger equation," J. Phys. A, 29, No. 20, 6581-6584 (1996).

11. M. Alsina and P. Bayer, Quaternion Orders, Quadratic Forms, and Shimura Curves, American Mathematical Society, Providence, RI (2004).

12. G. Shimura, Arithmetic and Analytic Theories of Quadratic Forms and Clifford Groups, American Mathematical Society, Providence, RI (2004).

13. D. W. Hoffmann and A. Lanhribi, "Quadratic forms and Pfister neighbors in characteristic 2," Trans. Amer. Math. Soc., No. 10, 40194052 (2004). 
14. A. M. Ateiwi, "A study of dichotomy of linear systems of difference equations using the quadratic forms," J. Fract. Calc., 25, 93-100 (2004).

15. F. Fang and J. Pan, "Secondary Brown-Kervaire quadratic forms and $\pi$-manifolds," Forum Math., 16, No. 4, 459-481 (2004).

16. T. Ueno, "Modular forms arising from zeta functions in two variables attached to prehomogeneous vector spaces related to quadratic forms," Nagoya Math. J., 175, 1-37 (2004).

17. W. K. Chan and M. Peters, "Quaternary quadratic forms and Hilbert modular surfaces," Contemp. Math., 344, 85-97 (2004).

18. W. Kohnen, "Special Siegel modular forms and singular series polynomials of quadratic forms," Contemp. Math., 344, 229-236 (2004).

19. A. Laghribi, "Quasi-hyperbolicity of totally singular quadratic forms," Contemp. Math., 344, 237-248 (2004).

20. R. Schulze-Pillot, "Representation by integral quadratic forms—a survey," Contemp. Math., 344, 303-321 (2004).

21. R. W. Fitzgerald and J. L. Yucas, "Pencils of quadratic forms over finite fields," Discrete Math., 283, 71-79 (2004).

22. M. Li and C. Dezhong, "Systems of Hermitian quadratic forms," Can. Math. Bull., 47, No. 1, 73-81 (2004).

23. M. Car, "Quadratic forms with polynomial coefficients," Acta Arithm., 113, No. 2, 131-155 (2004).

24. A. J. Bevelacqua, "Four dimensional quadratic forms over $F(X)$ where $I_{t}^{3} F(X)=0$ and a failure of the strong Hasse principle," Commun. Algebra, 32, No. 3, 855-877 (2004).

25. A. Teksan, "Representations of positive integers by a direct sum of quadratic forms," Results Math., 46, 146-163 (2004).

26. S. Jaschke, C. Keúppelberg, and A. Lindner, "Asymptotic behavior of tails and quantiles of quadratic forms of Gaussian vectors," J. Multivar. Anal., 88, No. 2, 252-273 (2004).

27. V. M. Bondarenko and A. M. Polishchuk, "On a criterion for the positive definiteness of one class of infinite quadratic forms," Nelin. Kolyvannya, 6, No. 1, 3-14 (2003).

28. V. M. Bondarenko, "On (min, max)-equivalence of posets and applications to Tits forms," Visn. Kyiv Univ., Ser. Fiz. Mat., No. 1, 24-25 (2005)

29. V. M. Bondarenko and M. V. Styopochkina, "On posets of width two with positive Tits form," Algebra Discrete Math., No. 2, 11-22 (2005). 